\title{
Primary cell-based phenotypic assays to pharmacologically and genetically study fibrotic diseases in vitro
}

\author{
S. Weigle, E. Martin, A. Voegtle, B. Wahl and M. Schuler
}

\begin{tabular}{|l|l|}
\hline Supplementary Information & Manuscript Section \\
\hline Figure S1 & $\begin{array}{l}\text { Results Section - TGFß-1-mediated fibroblast- } \\
\text { to-myofibroblast transition assay }\end{array}$ \\
\hline Figure S2 & $\begin{array}{l}\text { Results Section - TGFß-1-mediated epithelial-to- } \\
\text { mesenchymal transition assay }\end{array}$ \\
\hline Table S1 & $\begin{array}{l}\text { Methods Section - Fibroblast to myofibroblast } \\
\text { transitions assay }\end{array}$ \\
\hline Table S2 & $\begin{array}{l}\text { Methods Section - Epithelial to mesenchymal } \\
\text { transition assay }\end{array}$ \\
\hline
\end{tabular}




\section{Figure S1}
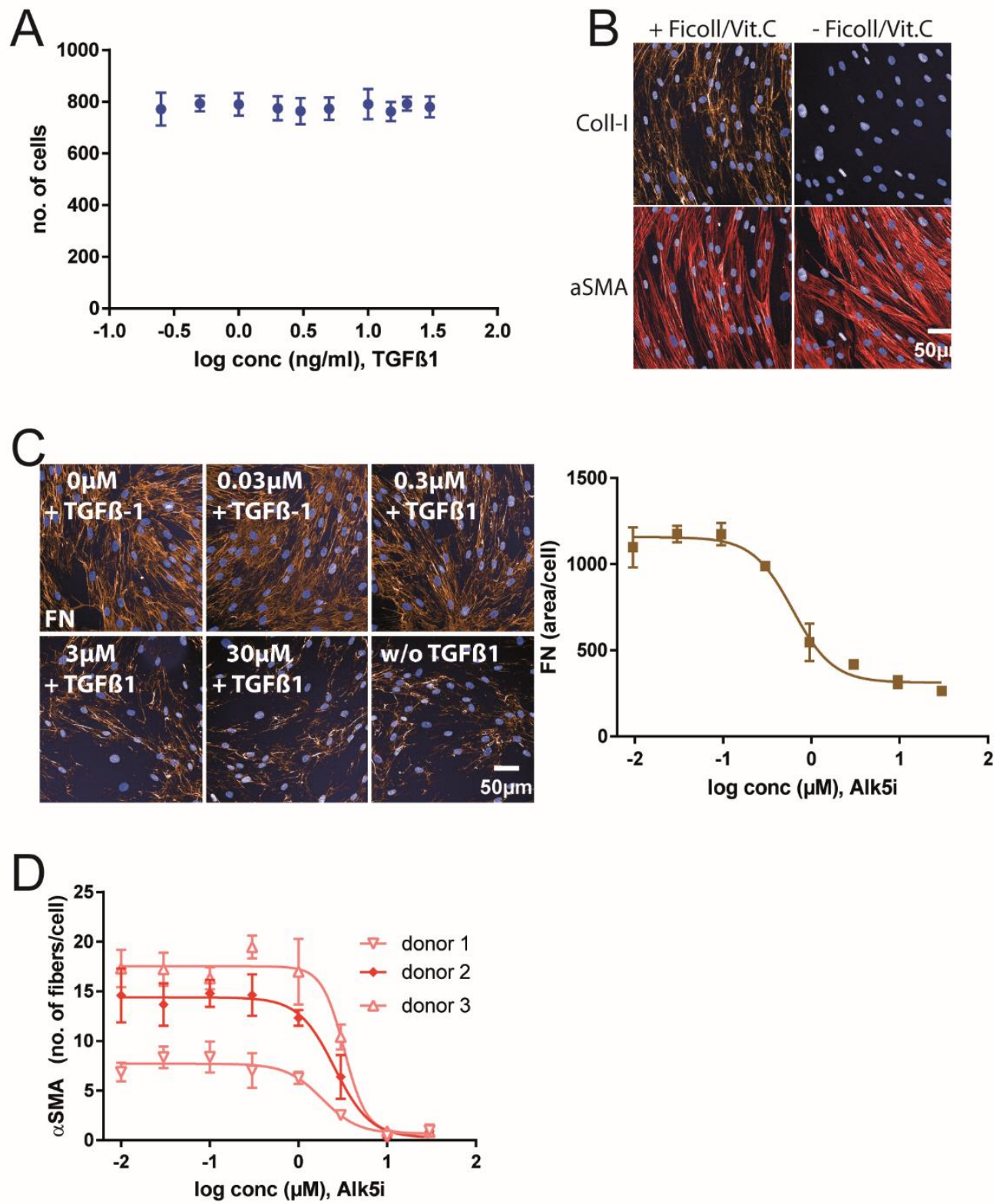

Figure S1. A. No effect of increasing TGFß-1 concentrations on cell counts in the FMT assay. B. Effect of the mixture of molecular crowding agent, vitamin-C, Ficoll-75 and -400 on QSMA and Coll-I expression. Only in the presence of the molecular crowding mixture Coll-I expression was detected. C. Images of NHLF cells (1000/well) treated with increasing doses of TGFB-1 and stained for nuclei, and fibronectin. Images were acquired with the IN Cell 2200 high content imager (right). Fibronectin area/cell was quantified with a custom protocol using Columbus software and plotted against increasing doses of TGFß-1. The Alki IC $\mathrm{C}_{50}$ was $0.6 \mu \mathrm{M}$ (left). D. Increasing Alk5i doses were added to different NHLF cell donors in the presence of $5 \mathrm{ng} / \mathrm{ml}$ TGFß-1. $\alpha$ SMA expression was quantified $72 \mathrm{~h}$ later. The potenty of the Alk5i was comparable for the 3 donors, but donor 1 showed a smaller maximal TGFß-1 effect. 


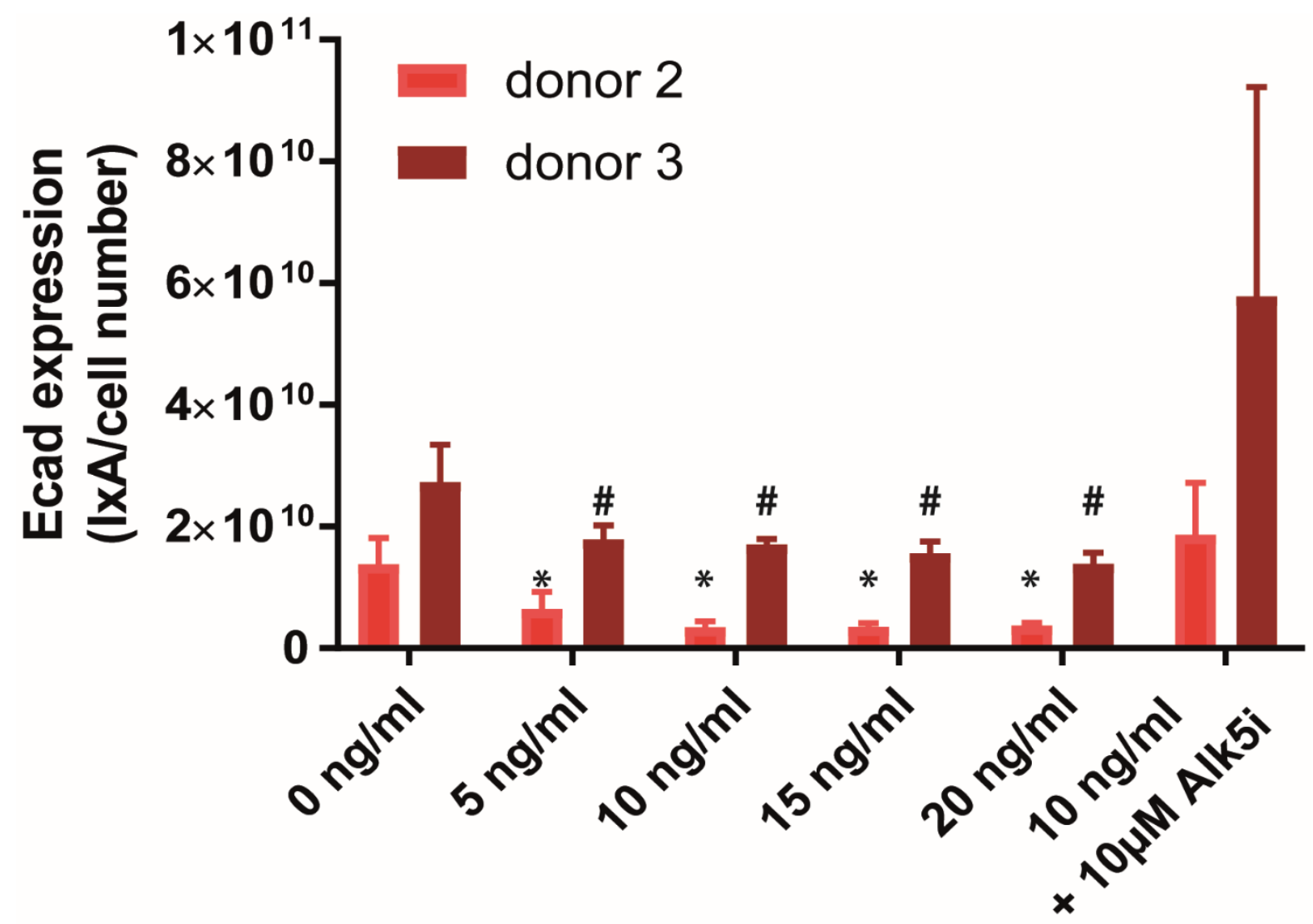

Figure S2. Increasing TGFB-1 doses and $10 \mathrm{ng} / \mathrm{ml}$ TGFB-1 plus $10 \mu \mathrm{M}$ of the Alki SB525334 were added to different hSAEC donors. Ecad expression was quantified $72 \mathrm{~h}$ later. TGFB-1 at a concentration of $10 \mathrm{ng} / \mathrm{ml}$ maximally inhibited Ecad expression, which was reversed by addition of an Alk5i. Compared to the other donor the assay window for the additional donors was smaller. 


\section{Table S1}
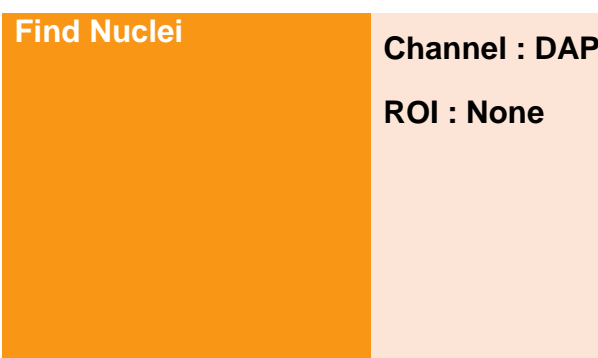

Channel : FITC2

Nuclei : Cells
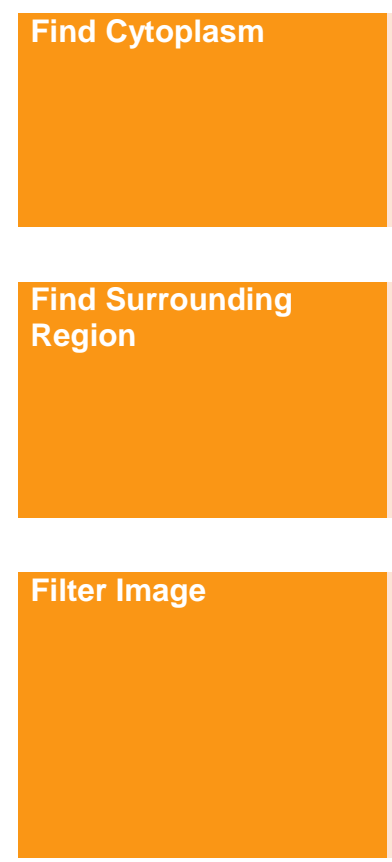

Common Threshold : 0.4

Area : $>30 \mu \mathrm{m}^{2}$

Split Factor : 7

Individual Threshold : 0.4

Contrast : $>0.1$

\section{Method : F}

Membrane Channel : Cy5

Individual Threshold : 0.1 (, numeric)

\section{Channel : FITC2 \\ Population : Cells \\ Region : Cell}

\section{Channel : Cy5}

Method : Texture SER

Filter : SER Ridge

Scale : $1 \mathrm{px}$

Normalization by : Region

Intensity

\section{Method : B}

Common Threshold : 0.2

Individual Threshold : 0.15

Include Input Region

\section{ABB: Find Simple Image Region}

Output Population :

Cells

\section{Channel : SER Ridge}

Threshold : 0.1

Output Population : Brights

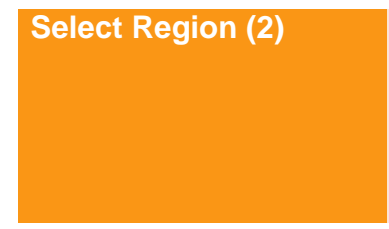

Population : Cells

Region : Full Cell

\section{Modify Population}

Method : Restrict by Mask

Population : Brights

Mask Region : Region
Output Region :

Full Cell

Output Image : SER Ridge
Output Region :

Candidate Fibers

\section{Population : Cells \\ Region : Candidate Fibers}

Method : Cluster by Distance

Distance : 0 px (fixed, menu)

Area : > 15 (, numeric) $\mathrm{px}^{2}$
Output Population : Candidates

Output Region : Region
Calculate Intensity Properties

\section{Channel : Cy5}

Population : Candidates

Region : Region

\section{Method : Standard}

Mean

Contrast
Output Properties :

Alexa 647 


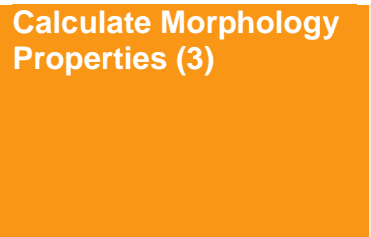

Population : Candidates

Method : Standard

Output Properties :

Region : Region

Area

length

Roundness

Length

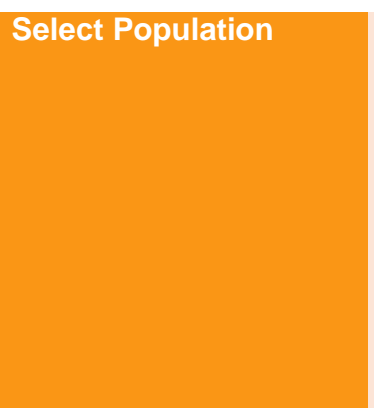

Population : Candidates

Method : Filter by Property

Alexa 647 Mean : > 300

Alexa 647 Contrast : > 0

length Length $[\mu \mathrm{m}]:>10$

length Roundness : $<=0.7$

Boolean Operations : F1 and

$\mathrm{F} 2$ and $\mathrm{F} 3$ and F4

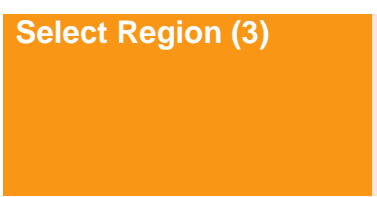

\title{
Population : Cells \\ Region : Full Cell
}

Method : Restrict by Mask

Output Region :

Population : Fibers

Mask Region : Region

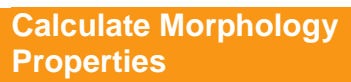

\section{Population : Cells \\ Region : Fibers}

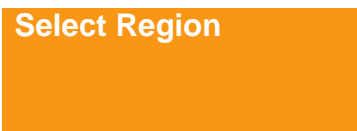

Population : Cells
Region : Fibers

\section{Calculate Morphology} Properties (2)

\author{
Population : Cells \\ Region : Fibers Skeleton
}

\section{Calculate Intensity}

Properties (2)

\section{Channel : Cy5}

Population : Cells

Region : Fibers

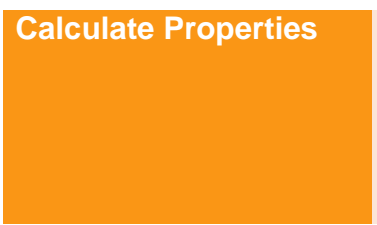

\section{Method : By Related Population}

Related Population : Fibers

Number of Fibers
Output Population : Fibers
Method : Standard

Output Properties : Fibers Skeleton

Area

Method : Standard

Output Properties :

Output Properties : Fibers

Output Regions : Fibers

Mean ABB: Find Simple
Image Region (2)
Channel : TexasRed

Threshold : 2000 (, numeric)

Output Population : Fibronectin 
Population : Collagen/Fibronectin

Region : Region

Area

Roundness

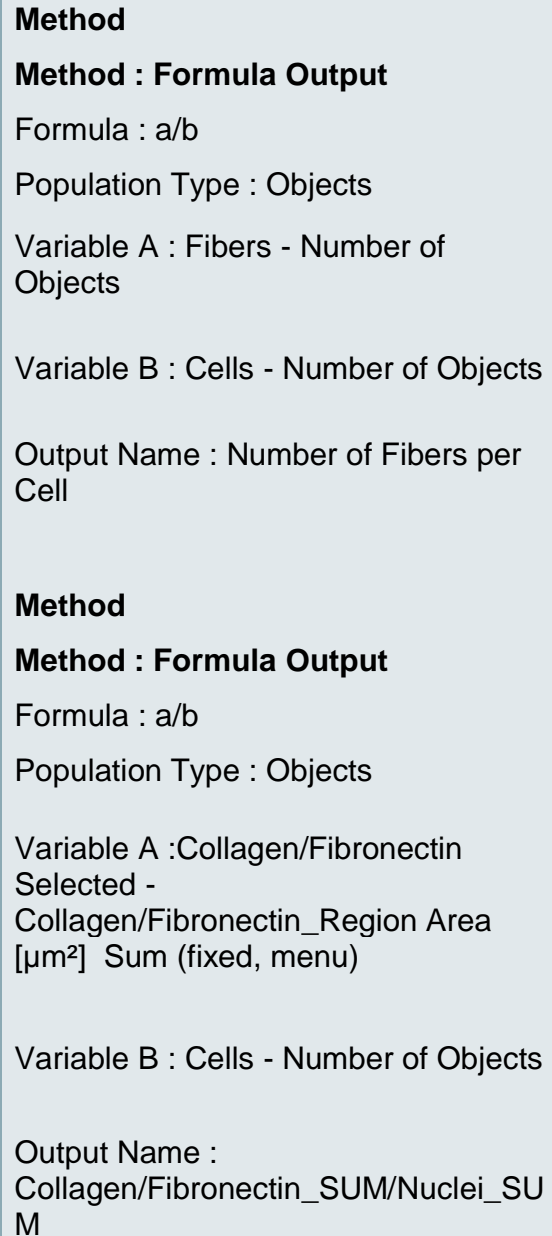

Output Properties Collagen/Fibronecti n_Region 
Table S2

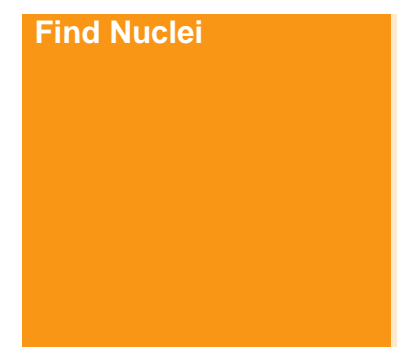

Channel : HOECHST 33342

ROI : None

Method : B

Common Threshold : 0.4

Area : $>30 \mu \mathrm{m}^{2}$

Split Factor : 7

Individual Threshold : 0.4

Contrast : $>0.1$

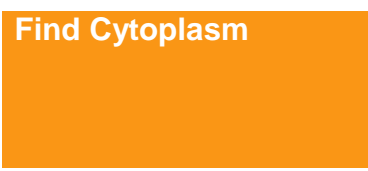

Channel : Alexa 488

Nuclei : Cells

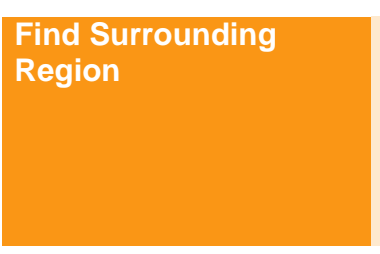

Channel : Alexa 647
Population : Cells
Region : Cell

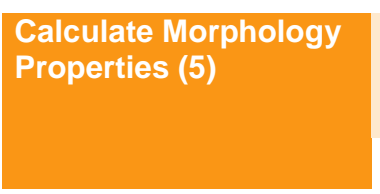

Population : Cells

Region : Full Cell

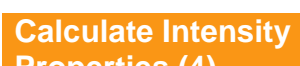

Properties (4)

\section{Method : F \\ Membrane Channel : Alexa 488 \\ Individual Threshold : 0.15}

\section{Method : B}

Common Threshold : 0.2

Individual Threshold : 0.15

Include Input Region

Method : Standard

Area

Roundness

Method : Standard

Mean
Output Population : Cells 


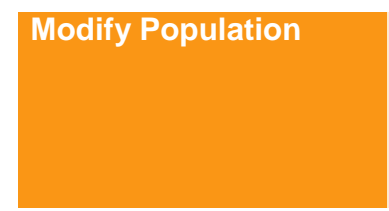

Population : Cells

Region : Candidate Ecad
Method : Cluster by Distance

Distance : $0 \mu \mathrm{m}$

Area : $>30 \mu \mathrm{m}^{2}$

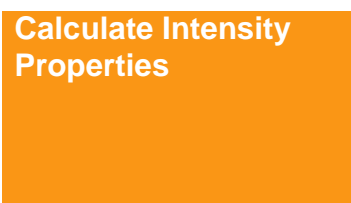

Calculate Morphology Properties (3)

\section{Ecad}

Channel : Alexa 647

Population : Candidates

Ecad

Region : Region

Population : Candidates

Region : Region

\section{Population : Candidates}

Ecad

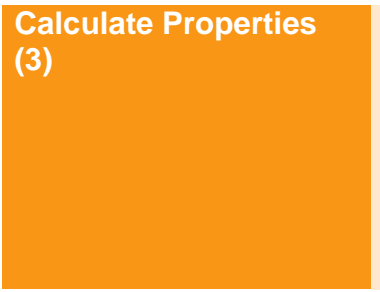

Mean

Sum
Method : Standard

Area

\section{Method : By Formula}

Formula : $A^{*} B$

Variable A : Alexa 647 Sum

Variable B : A-

Candidates_Ecad Area [ $\left.\mu^{2}{ }^{2}\right]$

Output Population :

Candidates Ecad

Output Region :

Region

Output Properties : Alexa 647

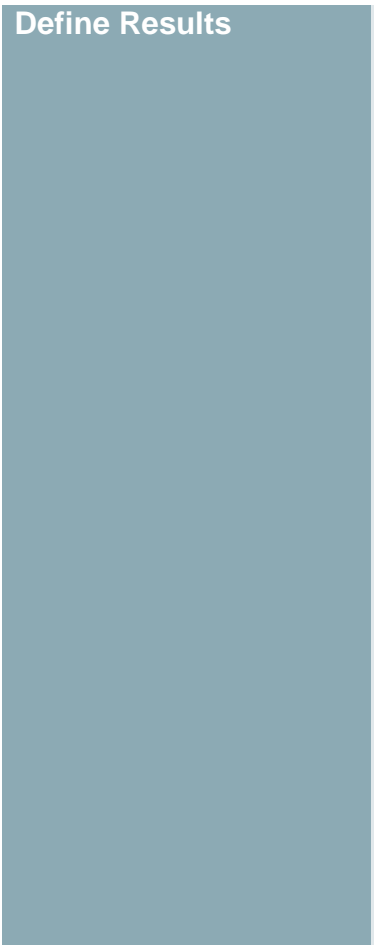
Population : Cells
Number of Objects
Method : Formula Output
Formula : $\mathrm{a}^{*} \mathrm{~b} / \mathrm{c}$
Population Type : Objects
Variable A : Candidates Ecad
- Alexa 647 Sum Sum
Variable B : Candidates Ecad
- A-Candidates_Ecad Area
$\left[\mu \mathrm{m}^{2}\right]$ Sum
Variable C : Cells - Number of
Objects
Output Name :
I Ecad Sum*A Ecad sum/c
ell number
Population : Ecad : None
Population : Cells : None
Population : Candidates
Ecad : None

Output Properties :

A-

Candidates_Ecad

Output Property :

IA_Candidates_E cad 